\title{
30. Assessment of dyspnoea in Covid 19 patients using modified Borg scale
} \section{Tsiafaki $^{1}$}

Vasileios Flampouraris ${ }^{1, *}$, Sotirios Kalinoglou ${ }^{1}$, Stella Kandreviotou ${ }^{1}$, Bogdan Raitsiou ${ }^{1}$, Maria Kokolaki ${ }^{1}$, Xanthi

${ }^{1}$ Sismanoglio-Amalia Fleming General Hospital of Athens, Department of Anaesthesiology and Pain Medicine, Athens, Greece.*vasflamp@gmail.com

Background: Dyspnoea (shortness of breath) is a common excruciating symptom. Shortness of breath is a feeling associated with impaired respiratory function. As difficulty breathing, I can not breathe. It occurs in $64 \%$ in Covid 19 patients.

Objective: Our goal is to evaluate dyspnoea using Borg scale that assesses the functional state of respiratory function.

Material-methods: We assessed (92) Covid 19 patients with a categorical ten-point rating, a corresponding verbal description (Table 1).

Table 1. 1-10 Borg Rating of Perceived Exertion Scale - Breathing Difficulty.

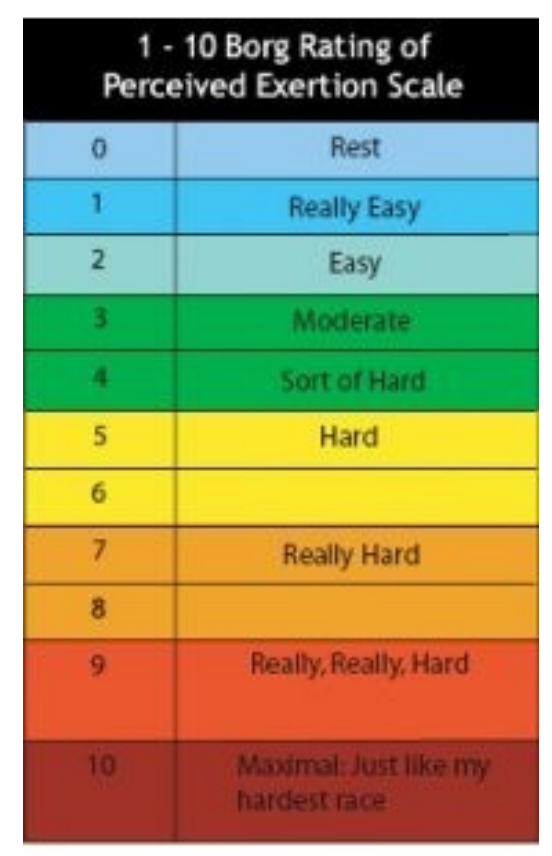

Patients were aged between 44-78 years old. Fifty of them (50) were obese, body weight $>$ greater than $90 \mathrm{~kg}$ with comorbidities (hypertension and diabetes mellitus). The assessment was performed by a specialist anaesthesiologist (pain clinic) on the Borg scale.

Results: (12) Covid 19 patients with moderate dyspnoea. (30) Covid 19 patients with severe dyspnoea (hard). (46) Covid 19 patients very severe dyspnoea (really hard). (4) Covid 19 patients maximum intolerable dyspnoea (maximum). We immediately intubated them. There's not internationally accepted way to assess shortness of breath. Scales are commonly used to assess respiratory difficulty (for example: respiratory distress observation scale).

Conclusions: The Borg scale has been used successfully in Covid 19 patients. Every patient experiences dyspnoea differently, depending on the severity of the disease. However, it seems that the assessment of dyspnoea on the Borg scale, taking into account co-factors leads to safer monitoring of severely ill Covid 19 patients.

\section{References}

[1] Multidisciplinary medicine 15 (1), 2020. 